\title{
lodine nutrition and prevalence of sonographic thyroid findings in adults in the Heilongjiang Province, China
}

\author{
Kuo Miao ${ }^{1 \wedge}$, Liwei Zhang ${ }^{1}$, Xiaoqiu Dong ${ }^{1}$, Yunfeng $\mathbf{Q i}^{1}$, Dejiao Kong ${ }^{1}$, Ying $\mathrm{Liu}^{2}$, Ning Zhao ${ }^{1}$, Jing $\mathrm{Hu}^{3}$ \\ ${ }^{1}$ Department of Ultrasound, The Fourth Affiliated Hospital of Harbin Medical University, Harbin, China; ${ }^{2}$ Key Laboratory of Etiology and \\ Epidemiology, National Health and Family Planning Commission, Center for Endemic Disease Control, Chinese Center for Disease Control and \\ Prevention, Harbin Medical University, Harbin, China; ${ }^{3}$ College of Bioinformatics Science and Technology, Harbin Medical University, Harbin, \\ China \\ Contributions: (I) Conception and design: All authors; (II) Administrative support: X Dong; (III) Provision of study materials or patients: All authors; \\ (IV) Collection and assembly of data: All authors; (V) Data analysis and interpretation: All authors; (VI) Manuscript writing: All authors; (VII) Final \\ approval of manuscript: All authors. \\ Correspondence to: Xiaoqiu Dong. Department of Ultrasound, The Fourth Affiliated Hospital of Harbin Medical University, Harbin 150001, \\ Heilongjiang, China. Email: Dongxq0451@163.com.
}

\begin{abstract}
Background: Thyroid disease and thyroid nodules are common clinical problems. Iodine nutrition plays an important role in thyroid disease evolution. Here, we aimed to estimate the iodine nutritional status and prevalence of thyroid disease in the adults of the Heilongjiang Province in northeast China.

Methods: We performed a cross-sectional ultrasound (US)-based survey on volunteers aged 2070 years from 30 regions of the Heilongiiang Province. The participants were recruited using the probability proportional to size (PPS) method, and consent for US screening was obtained from them. The survey was performed by trained technicians using the same US equipment with a 6-15 MHz linear transducer (MyLab $30 \mathrm{cv}$, Italy) and was hosted in public community locations such as local hospitals and outpatient departments. Information on basic demographic characteristics, such as urinary iodine and iodine intake were collected. The age- and sex-adjusted prevalence of thyroid disease was determined through direct standardization and reported using the province's population in 2016 as reference.

Results: From December 12, 2017, to March 10, 2019, 3,754 participants with a mean age of $48.65( \pm 12.39)$ years participated in the study. Of them, 3,643 had reliable urinary iodine data. The median urinary iodine and salt iodine concentrations within the normal range were $163.30 \mu \mathrm{g} / \mathrm{L}$ and $24.30 \mathrm{mg} / \mathrm{kg}$, respectively. The age- and sex-adjusted prevalence of thyroid disease was $52.91 \%$. Diffuse thyroid disease (DTD), focal thyroid lesions (FTL), and coexistence of both diseases were prevalent in 8.68\%, 36.58\%, and $7.65 \%$ of the participants, respectively. The prevalence of the five categories according to US-based survey features in the ACR TI-RADS (i.e., TR1, TR2, TR3, TR4, and TR5) was 7.71\%, 14.53\%, 3.44\%, 14.82\%, and $3.51 \%$, and the prevalence of nodules that needed fine-needle aspiration was $2.55 \%$.

Conclusions: In Heilongjiang Province, adults aged 20-70 years belong to the optimal iodine status. Further, the salt iodine levels are in the normal range. Thyroid diseases are highly prevalent in this age group; however, the intervention rate is low. We provided population-based estimates of the prevalence of thyroid disease and the iodine status in adults of Heilongjiang Province. These findings are useful to support effective intervention planning for thyroid disease.
\end{abstract}

Keywords: Prevalence; thyroid; ultrasound (US); iodine nutrition; salt iodine

Submitted Feb 01, 2020. Accepted for publication Aug 21, 2020.

doi: 10.21037/atm-20-1152

View this article at: http://dx.doi.org/10.21037/atm-20-1152

\footnotetext{
$\wedge$ ORCID: 0000-0001-6985-3253.
} 


\section{Introduction}

Iodine nutrition is a key determinant of thyroid disease worldwide $(1,2)$. More than $90 \%$ of dietary iodine eventually appears in the urine. Thus, the urinary iodine concentration (UIC) is an excellent biomarker of recent iodine intake (2). The iodine status of the general population in China has been optimal in recent years, with an average UIC of $197 \mu \mathrm{g} / \mathrm{L}$ (3). In 2012, a new national decree (GB-268782011) for salt iodization was launched. The standard SIC was adjusted to $20-30 \mathrm{mg} / \mathrm{kg}$, within $\pm 30 \%$ of the recommended level for the provincial governments (4).

Thyroid disease is a common clinical problem, and thyroid nodules are also exceedingly common. They are prevalent in $28.8 \%$ of the population in China (5) and up to $68 \%$ of adults in Germany (6). High-resolution ultrasound (US) is generally considered the standard modality for evaluating thyroid size and morphology (7-9). The ACR thyroid imaging, reporting, and data system (TI-RADS) provide guidance for the management of thyroid nodules based on their US appearance (10). Thyroid disease also includes diffuse types, and several previous studies have suggested that diffuse thyroid disease (DTD) could be diagnosed using US (11-13). Iodine nutrition is a major risk factor of thyroid disease (1). U-shaped curves for the relationship between iodine intake and the risk of thyroid disease are common (14).

Heilongjiang Province is located in the northeast region of China. The people consume a salt-rich diet. Thus, it is unknown whether the iodine status is adaptive. The prevalence of US-based thyroid disease in Heilongjiang Province is also unknown. Therefore, this study aimed to estimate the iodine status and prevalence of thyroid disease through US screening and the assess the relationship between them in adults of Heilongjiang Province, China.

We present the following article in accordance with the SURGE reporting checklist (available at http://dx.doi. org/10.21037/atm-20-1152).

\section{Methods}

\section{Study design and survey organization}

This study was a cross-sectional US-based survey that recruited volunteers aged 20-70 years in Heilongjiang Province. The study was conducted in accordance with the Declaration of Helsinki (as revised in 2013). The study was approved by institutional ethics board of the fourth affiliated hospital of Harbin Medical University (No.:
2020-SCILLSC-07) and informed consent was taken from all the patients.

A cluster sampling technique, probability proportional to size (PPS) sampling, in which the probability that a particular sampling unit will be selected in the sample is proportional to the sampling unit, was employed in the present study. In the first stage, with a county or district as the sampling unit, 30 counties and districts were selected from 110 counties and districts in Heilongjiang Province (Figure 1). One urban residential community or rural village community was then randomly selected from each county or district via PPS. Finally, approximately 120 people aged 20-70 years from each community or village were selected. We stratified the final sample by sex and age distribution based on the 2016 Heilongiiang statistical yearbook.

We included only those living in their current residence for at least one year in the sampling frame. Furthermore, we excluded individuals who consumed iodine-rich foods within three days before the US, those who could not complete the questionnaire or those who were pregnant or breastfeeding.

Spot urine and salt samples were delivered to the Institute of Iodine Deficiency Disease Control and Prevention, China Center for Disease Control and Prevention, Harbin Medical University for measuring UIC and SIC. UIC was determined using the modified acid digestion method. Briefly, this method is used to determine UIC using As3+-Ce4+catalytic spectrophotometry (WS/ T 107-2016, Ministry of Health of the People's Republic of China). SIC was analyzed using the general test method in the salt industry (GB/T 130257.7-2012, Ministry of Health of the People's Republic of China). Results of the questionnaire survey and US screening were included in the analysis of thyroid disease prevalence. Participants with no urinary iodine data owing to urine sample loss or urine sample contamination were excluded in the urine iodine analysis.

\section{Survey and US screening protocol}

The volunteers were initially interviewed before thyroid screening. Trained interviewers administered a standardized questionnaire that included information on demographic characteristics, smoking or drinking status, medical history, history of hypertension or diabetes, and other risk factors for thyroid disease.

We collected the urine and sodium chloride of each volunteer. The subjects were instructed to use a urine cup to 


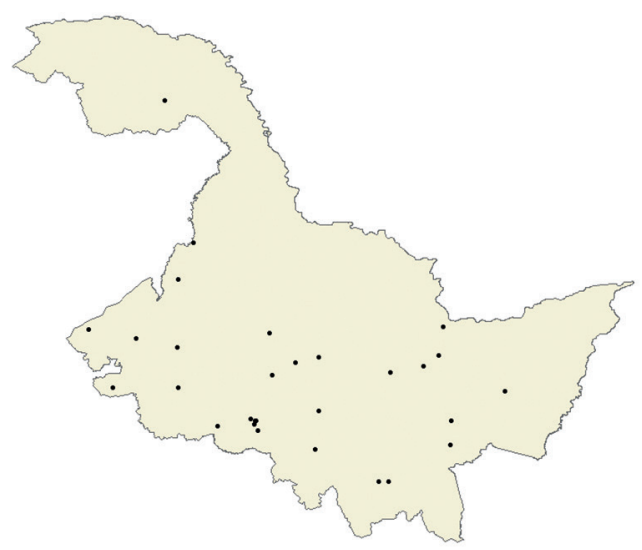

Figure 1 Distribution of the 30 counties and districts in Heilongjiang province investigated in this study.

collect their urine, pour it into the barrel, and screw down the lid. Samples were then numbered and immediately placed in a $4{ }^{\circ} \mathrm{C}$ refrigerator. The salt was put into the selfsealing bag and marked with the corresponding number.

US scans were performed by clinicians working in the Department of Ultrasound, the Fourth Affiliated Hospital of Harbin Medical University, who had at least five years of experience in the US diagnosis of thyroid disease. Before the survey, all doctors received standardized training and orientation to standardized diagnostic criteria. Thyroid ultrasonography was performed to evaluate the thyroid gland using the same portable US equipment with a 6-15 MHz linear transducer (MyLab $30 \mathrm{cv}$, Italy), with the subject lying supine and the neck hyperextended.

First, an axial scan of the whole gland and the isthmus was conducted, and then the width of each lobe and anteroposterior diameters were measured. Consequently, a longitudinal scan through each lobe was conducted, and the lobe length was measured. The thyroid gland was then identified to be either normal, with DTD, or with characteristics of focal thyroid lesions (FTL). Ultrasonography findings indicative of DTD are characterized by a diffusely enlarged or normal size thyroid gland or small size in later stages of DTD, decreased or increased diffuse heterogeneous parenchymal echogenicity, a coarse echotexture, and micronodulation. For the FTL, the clinicians measured the main lesions of the same composition and recorded the composition, echogenicity, shape, margin, echogenic foci, and the vascular pattern of the nodules. Then, they classified the nodules according to ACR TI-RADS guidelines.

\section{Statistical analysis}

The demographic characteristics of the study population were described as numbers and percentages. The overall prevalence of thyroid disease was adjusted with direct standardization by sex and age group based on the province's population in 2016 as a reference. We estimated the prevalence of thyroid disease for both men and women in five age groups (i.e., 20-29, 30-39, 40-49, 50-59, and $60-70$ years) in rural and urban settings. We also estimated the prevalence of thyroid disease for different UICs. Furthermore, we estimated the prevalence of DTD, FLT, and both DTD and FLT and compared their relationship with UICs. For the prevalence calculation, the data containing personal information and US data, and for the calculation of median urinary iodine, the data should also include urinary iodine data. Otherwise the data was not included in the research analysis. Differences in the prevalence of thyroid disease were assessed using the Pearson's $\chi^{2}$ test corrected for the sampling design with the second-order correction of Rao and Scott and converted into an F-statistic. The test was performed with the observed and expected frequencies calculated by weighting data according to the relative age and sex distribution of the reference population. All estimates were presented with $95 \%$ confidence intervals (CIs) calculated with the logit transformation and accounting for the increased variance because of the sampling design through the Taylor linearization method. All statistical analyses were performed using R version 3.5.1 (http://www.R-project.org). A two-sided $\mathrm{P}$ value of $<0.05$ was considered statistically significant.

\section{Results}

Between December 12, 2017 and March 10, 2019, 3,754 individuals attended the study sessions. Of them, 3,754 (1,147 men and 2,607 women) had basic information and US data, and 3,643 had urine iodine and salt iodine data. The general patient characteristics and risk factors and iodine nutritional status are presented in Table 1.

The median UICs was $163.30 \mu \mathrm{g} / \mathrm{L}$ for the overall population; $173.23 \mu \mathrm{g} / \mathrm{L}$ in men, $159.34 \mu \mathrm{g} / \mathrm{L}$ in women, $163.04 \mu \mathrm{g} / \mathrm{L}$ in urban areas, and $169.76 \mu \mathrm{g} / \mathrm{L}$ in suburban areas. Meanwhile, it was $208.73,178.84,163.30,155.78$, and $149.70 \mu \mathrm{g} / \mathrm{L}$ in those aged 20-29, 30-39 40-49, 50-59, and $60-70$ years, respectively. In total, $25.57 \%, 38.13 \%$, and $37.30 \%$ of urine samples had a median UIC of $<100$, 
Table 1 Patient characteristics according to sonogram findings of the thyroid gland

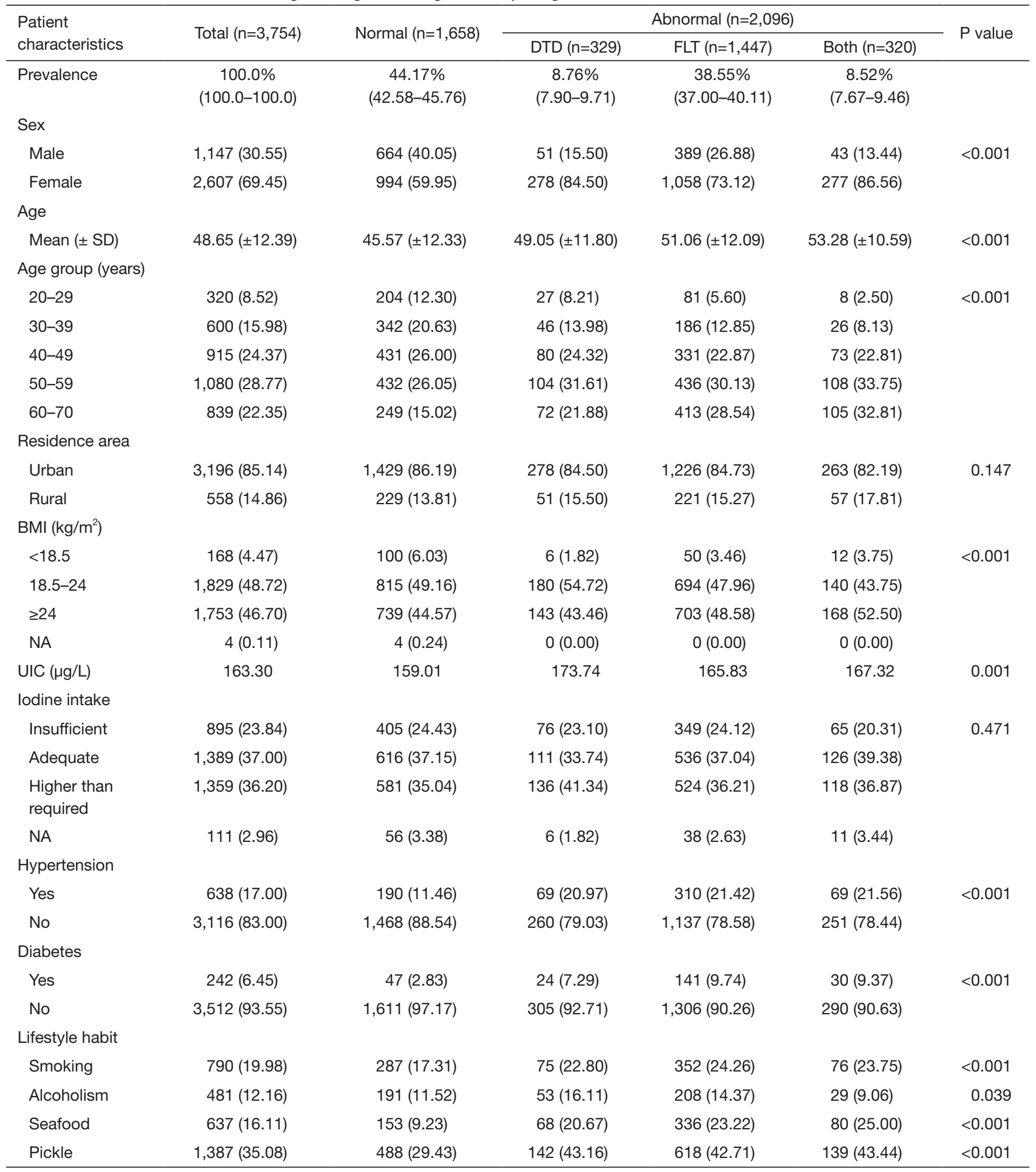

Data are presented as $\mathrm{n}(\%)$ or \% $(95 \% \mathrm{Cl})$. Standardized prevalence is directly standardized by age and sex using the Heilongjiang Province's population in 2016 as the reference. DTD, diffuse thyroid disease; FLT, focal thyroid lesions; Both, coexistence of DTD and FLT; $\mathrm{BMI}$, body mass index; UIC, urinary iodine concentration; NA, not available. 
$100-200$, and $\geq 200 \mu \mathrm{g} / \mathrm{L}$, respectively. These were $21.12 \%$, $39.75 \%$, and $39.13 \%$ in men and $26.10 \%, 37.41 \%$, and $36.49 \%$ in women $(\mathrm{P}=0.005$ for sex difference, Table 2). The median SIC was $24.30 \mathrm{mg} / \mathrm{kg}$ within the normal range.

The overall age-standardized prevalence of thyroid disease was $52.91 \%$ (95\% CI: 50.58-55.34\%) among the general population aged 20 to 70 years. The prevalence was higher in women than in men [58.91\% (95\% CI: $55.93-$ 62.06\%) vs. $39.61 \%$ (95\% CI: $36.05-43.47 \%$ ), $\mathrm{P}<0.001$ ]. This sex difference was noted across all age groups. The age-standardized prevalence of thyroid disease by DTD, FLT, and both was $8.68 \%, 36.58 \%$, and $7.65 \%$ in the overall population; $3.91 \%, 31.95 \%$, and $3.74 \%$ in men; and $10.87 \%, 38.71 \%$, and $9.33 \%$ in women, respectively. For FLT, the prevalence of the five categories of US features (i.e., composition, echogenicity, shape, margin, and echogenic foci) in ACR TI-RADS (i.e., TR1, TR2, TR3, TR4, and TR5) was $6.38 \%, 13.38 \%, 2.19 \%, 10.49 \%$, and $3.05 \%$ in men and $8.37 \%, 15.02 \%, 3.98 \%, 16.70 \%$, and $3.74 \%$ in women. The prevalence of nodules requiring fine-needle aspiration (FNA) was $2.03 \%$ in men and $2.81 \%$ in women (Figure 2).

The age-standardized prevalence of thyroid disease in the insufficient, the adequate, and the above normal iodine intake groups was $49.35 \%, 52.35 \%$, and $55.76 \%$, respectively. For DTD, the prevalence was $8.24 \%, 7.81 \%$, and $9.94 \%$, respectively, while that for FLT was $34.86 \%$, $36.72 \%$, and $37.60 \%$, respectively. For both, the prevalence was $6.24 \%, 7.82 \%$, and $8.22 \%$, respectively. The prevalence of DTD in UIC group above $300 \mu \mathrm{g} / \mathrm{L}$ was higher than the group $100-199 \mu \mathrm{g} / \mathrm{L}$ in all population $(\mathrm{P}=0.02)$ (Figure 3).

The distribution of urine iodine and the prevalence of thyroid disease in Heilongjiang Province is presented in Figure 4. For most areas, the UIC was in the normal range, but the UIC in Daqing City was $213.34 \mu \mathrm{g} / \mathrm{L}$. Thyroid disease was most prevalent in Jiamusi City at a rate of $66.67 \%$ (95\% CI: $61.00-71.89 \%$ ), whereas it was least prevalent in Hegang City at a rate of $30.08 \%$ (95\% CI: $22.66-38.71 \%)$. The distribution of salt urine within normal limits, with the highest at $26.50 \mathrm{mg} / \mathrm{kg}$ and the lowest at $22.20 \mathrm{mg} / \mathrm{kg}$.

\section{Conclusions}

Thyroid disease is a global health problem that can substantially impact well-being, and iodine nutrition remains a key determinant of thyroid function worldwide (1). To the best of our knowledge, there are no recent data about thyroid disease and iodine nutrition in the adult population aged 20-70 years of the Heilongiiang Province, which is located in the northeast of China. Thus, our findings fill several knowledges lacunae about the thyroid disease epidemic and iodine nutrition in China. First, the result of our study shows that the median UIC of adults aged 20-70 years in Heilongjiang Province was $163.30 \mu \mathrm{g} / \mathrm{L}$. This is considered as optimal iodine status by the World Health Organization (WHO), United Nations International Children's Emergency Fund (UNICEF), and International Council for Control of Iodine Deficiency Disorders (ICCIDD) (15). Second, our data indicate that $52.91 \%$ of the general adult population (or 15.71 million adults) aged 20-70 years in Heilongjiang Province had thyroid disease. Third, to our best knowledge, we report for the first time the prevalence of FTL according to the ACR TI-RADS guideline and the prevalence of nodules that needed FNA in Heilongjiang Province.

A previous study showed that more than $90 \%$ of dietary iodine eventually appears in the urine, and thus the UIC is an excellent biomarker of recent iodine intake (2). However, the UIC could only be used to assess the iodine status of a population, but not of the individual in that population (16). In this study, the median UIC of adults aged 20-70 years in Heilongjiang Province was $163.30 \mu \mathrm{g} / \mathrm{L}$. According to the WHO/UNICEF/ICCIDD (15), the level was the optimal iodine status. In those aged 20-29 years, the UIC was $208.73 \mu \mathrm{g} / \mathrm{L}$, which higher than the required iodine intake amount. However, this needs to be further verified. The UIC was $95.39 \mu \mathrm{g} / \mathrm{L}$ in Heilongjiang Province in 1995 (17), which is in accordance with the prevalence of iodine deficiencies in China until the introduction of USI in 1995. Since 2009, China's government has been considering lowering the salt iodization standard from the then mandatory mean of $35 \mathrm{mg} / \mathrm{kg}$. In 2012, China's government decided that while the USI remains mandatory, provinces may now iodize salt to a median within the range of $20-30 \mathrm{mg} / \mathrm{kg}$ depending on iodized salt coverage and UIC (18). In our study, the median SIC was $24.30 \mathrm{mg} / \mathrm{kg}$ in Heilongjiang Province, which is within the normal range.

Many cross-sectional surveys have reported the prevalence of thyroid disease in various Chinese regions (14,19-21). Zou et al. (19) evaluated 7,904 participants in Shanghai and reported a $36.61 \%$ prevalence rate of thyroid disease in the population aged 5-69 years. In Xinjiang province, an inland region in China, thyroid nodules were reported to be prevalent in $27.72 \%$ of individuals aged older than 18 years (20). In our study, thyroid disease and thyroid 
Table 2 Characteristics of the study population and thyroid disease by iodine intake

\begin{tabular}{|c|c|c|c|c|c|}
\hline Characteristics & Total $(n=3,643)$ & Insufficient $(n=895)$ & Adequate $(n=1,389)$ & Higher than required $(n=1,359)$ & $P$ value \\
\hline \multicolumn{6}{|l|}{ Sex } \\
\hline Male & $1,122(30.80)$ & $237(26.48)$ & $446(32.11)$ & $439(32.30)$ & 0.005 \\
\hline \multicolumn{6}{|l|}{ Age } \\
\hline Mean (SD) & $48.65( \pm 12.39)$ & $50.88( \pm 11.52)$ & $48.92( \pm 12.26)$ & $46.92( \pm 12.82)$ & $<0.001$ \\
\hline 20-29 & $310(8.51)$ & $47(5.25)$ & $102(7.34)$ & $161(11.85)$ & $<0.001$ \\
\hline 30-39 & $585(16.06)$ & $106(11.84)$ & $243(17.50)$ & $236(17.37)$ & \\
\hline $40-49$ & 885 (24.29) & $214(23.91)$ & $325(23.40)$ & $346(25.46)$ & \\
\hline $50-59$ & $1,049(28.79)$ & $283(31.62)$ & $413(29.73)$ & $353(25.97)$ & \\
\hline Rural & $531(14.58)$ & $119(13.30)$ & $203(14.61)$ & $209(15.38)$ & \\
\hline \multicolumn{6}{|l|}{$\mathrm{BMI}\left(\mathrm{kg} / \mathrm{m}^{2}\right)$} \\
\hline$<18.5$ & $158(4.34)$ & $46(5.14)$ & $58(4.18)$ & $54(3.97)$ & $<0.001$ \\
\hline $18.5-24$ & $1,765(48.45)$ & $480(53.63)$ & $671(48.31)$ & $614(45.18)$ & \\
\hline$\geq 24$ & $1,716(47.10)$ & $368(41.12)$ & $659(47.44)$ & $689(50.70)$ & \\
\hline NA & $4(0.11)$ & $1(0.11)$ & $1(0.07)$ & $2(0.15)$ & \\
\hline \multicolumn{6}{|l|}{ Disease history } \\
\hline Hypertension & $604(16.58)$ & $148(16.54)$ & $229(16.49)$ & $227(16.70)$ & 0.988 \\
\hline Pickle & $1,354(37.17)$ & $332(37.09)$ & $480(34.56)$ & $542(39.88)$ & 0.015 \\
\hline \multicolumn{6}{|c|}{ Sonogram findings of the thyroid gland } \\
\hline Normal & $1,602(43.97)$ & $405(45.25)$ & $616(44.35)$ & $581(42.75)$ & 0.471 \\
\hline DTD & $323(8.87)$ & $76(8.49)$ & $111(7.99)$ & $136(10.01)$ & \\
\hline FLT & $1,409(38.68)$ & $349(39.00)$ & $536(38.59)$ & $524(38.56)$ & \\
\hline Both & $309(8.48)$ & $65(7.26)$ & $126(9.07)$ & $118(8.68)$ & \\
\hline \multicolumn{6}{|l|}{ ACR TI-RADS } \\
\hline 1 & $261(7.16)$ & $53(5.92)$ & $99(7.13)$ & $109(8.02)$ & 0.311 \\
\hline 2 & 585 (16.06) & $156(17.43)$ & 219 (15.77) & $210(15.45)$ & \\
\hline 3 & 143 (3.93) & 33 (3.69) & $50(3.60)$ & $60(4.41)$ & \\
\hline 4 & $584(16.03)$ & 135 (15.08) & 233 (16.77) & 216 (15.89) & \\
\hline 5 & 136 (3.73) & $37(4.13)$ & $57(4.10)$ & 42 (3.09) & \\
\hline
\end{tabular}

Data are presented as $\mathrm{n}$ (\%). Standardized prevalence is directly standardized by age and sex using the Heilongjiang Province's population in 2016 as the reference. DTD, diffuse thyroid disease; FLT, focal thyroid lesions; Both, coexistence of DTD and FLT; BMI, body mass index; UIC, urinary iodine concentration; NA, not available. 

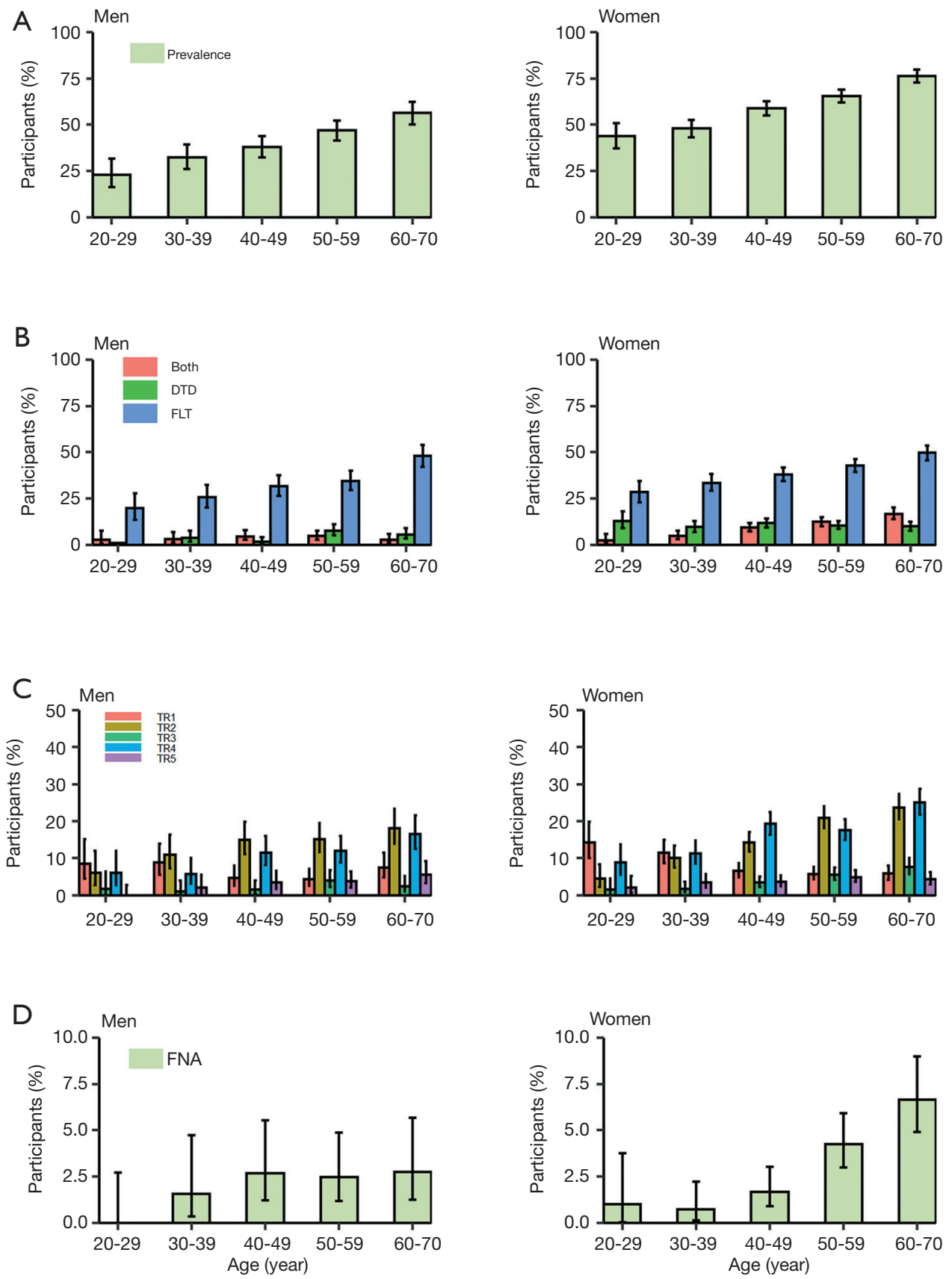

Figure 2 Overall prevalence of thyroid disease; prevalence of thyroid disease by DTD, FLT, and both DTD and FLT; prevalence of ACR TI-RADS categories of thyroid nodules; and prevalence of FNA among the male and female study participants. Data shown are stratified by age and sex. (A) Overall prevalence of thyroid disease. (B) Prevalence of thyroid disease by DTD, FLT, and both. (C) Prevalence of ACR TIRADS categories of thyroid nodules. (D) Prevalence of FNA. DTD, diffuse thyroid disease; FLT, focal thyroid lesions; Both, coexistence of DTD and FLT; FNA, fine-needle aspiration. 

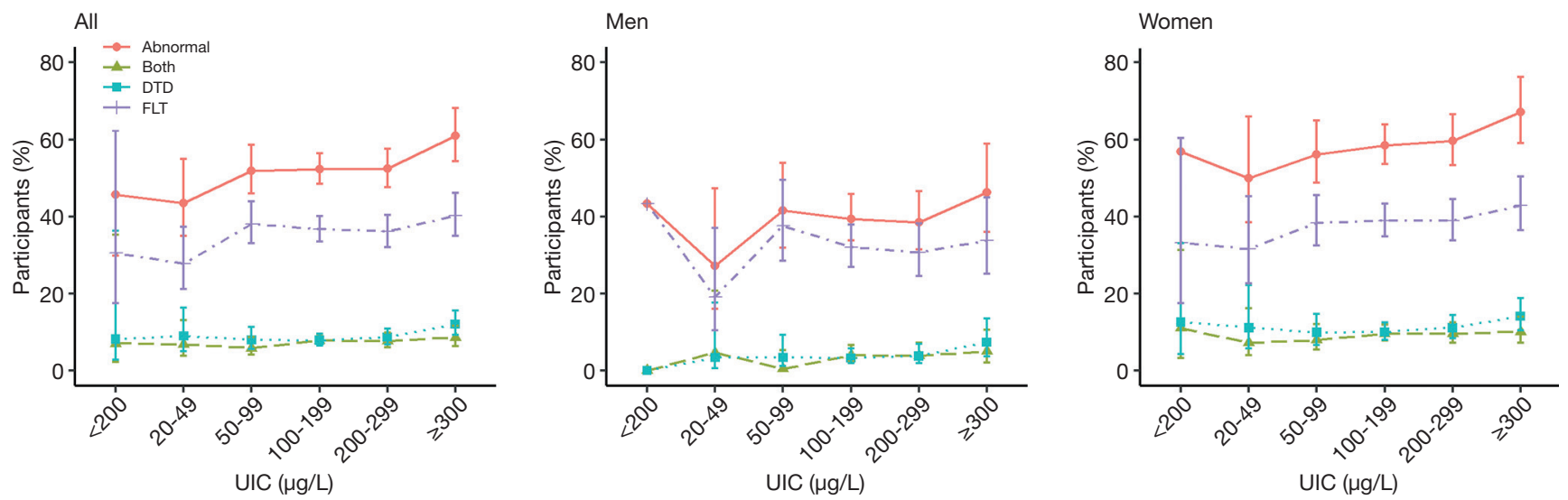

Figure 3 Relationship between the prevalence of thyroid disease and the UIC. Data shown are stratified by age and sex. DTD, diffuse thyroid disease; FLT, focal thyroid lesions; Both, coexistence of DTD and FLT; UIC, urinary iodine concentration.
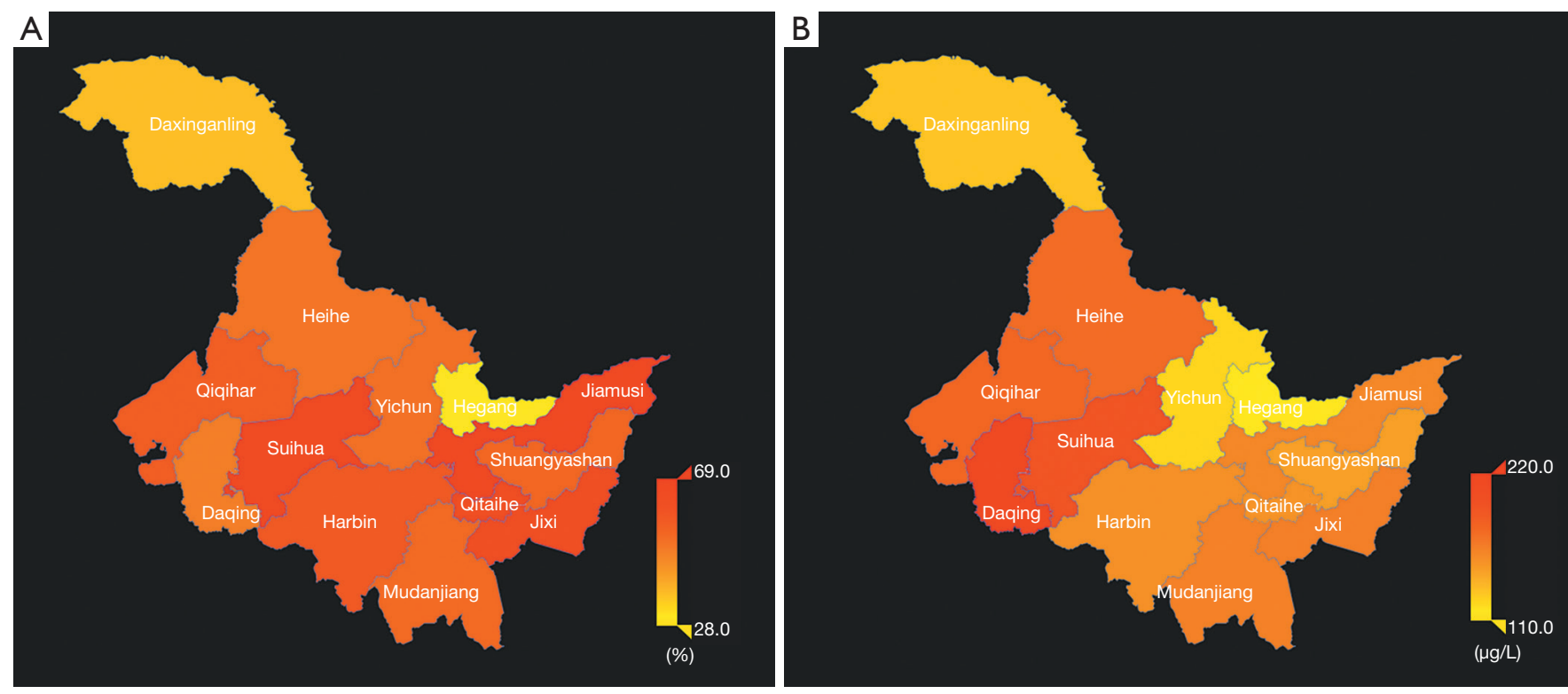

Figure 4 Distribution of the prevalence of thyroid disease (A) and urine iodine (B) in Heilongjiang Province.

nodules were prevalent in $52.91 \%$ (39.61\% for men and $58.91 \%$ for women) and $36.58 \%$ of adults aged $20-70$ years, respectively, in Heilongjiang Province. This was significantly higher than those reported in other provinces in China. However, this was consistent with the observations made by Guth et al., who used high-resolution US to report a prevalence of up to $53 \%$ in adults (6). The variation could be due to the difference in diagnostic criteria between previous studies in China and our study. However, to our knowledge, there were no data about the prevalence of thyroid disease in Heilongiiang Province, and thus we cannot compare the prevalence trends in this area.

In 2017, the American College of Radiology issued the White Paper of the ACR TI-RADS Committee (10), and this is by far the most authoritative guideline for thyroid nodules. In this study, we estimated the prevalence of thyroid nodules that needed FNA in Heilongiiang Province according to the ACR TI-RADS Committee guidelines. The prevalence rate was $2.55 \%$, which is far below the prevalence of the overall FLT at $36.58 \%$. This indicates that thyroid nodules are highly prevalent, but the rate of intervention is low.

Our analysis of the relationship between UIC and thyroid 
disease showed that the prevalence of DTD in UIC group above $300 \mu \mathrm{g} / \mathrm{L}$ was higher than the group 100-199 $\mu \mathrm{g} / \mathrm{L}$ in all population. However, the median UICs were not significantly different between those with normal thyroid and thyroid disease. Thyroid disease and UIC showed a U-shaped association in the study by Song et al. (14). The difference may be because of the different diagnostic criteria. Song et al. used blood test to diagnose thyroid disease, whereas we used high-resolution US.

The UIC is within the normal range in most areas in Heilongiiang Province. However, the UIC in Daqing City was higher than required. And thus, a further survey is needed to determine whether salt iodine should be adjusted or not.

Our study has several limitations. First, women and older people were oversampled because many men and younger people (migrant workers) were working outside their permanent residential regions. However, all estimates were weighted to correct for oversampling and nonresponses in the study. Second, the study did not include subjects aged below 18 years. Third, this was a crosssectional study and hence, did not evaluate individual changes over time. Furthermore, since blood testing is an invasive examination, the thyroid was only evaluated using US, and the levels of thyroid serum thyrotropin, free thyroxine (T4), free triiodothyronine (T3), thyroid peroxidase antibody, and thyroglobulin antibody were not measured. Fourth, the UIC was evaluated using random urine iodine that reflected the iodine status of the region, but not the individual iodine status. A follow-up study to determine the incidence rate of thyroid diseases and the relationship between the prevalence of thyroid diseases and individual iodine status is needed.

In conclusion, our data indicate that in Heilongjiang Province, adults aged 20-70 years are belonging to the optimal iodine status. Further, the salt iodine levels are within the normal range. Thyroid diseases are highly prevalent in this age group, but the rate of intervention is low.

\section{Acknowledgments}

We thank the participants of the survey. We thank the organizers of each district. We would like to thank Editage (www.editage.cn) for English language editing.

Funding: This work was supported by the Natural Science Foundation of Heilongjiang Province, China (Grant No. ZD2017016).

\section{Footnote}

Reporting Checklist: The authors have completed the SURGE reporting checklist. Available at http://dx.doi. org/10.21037/atm-20-1152

Data Sharing Statement: Available at http://dx.doi. org/10.21037/atm-20-1152

Conflicts of Interest: All authors have completed the ICMJE uniform disclosure form (available at http://dx.doi. org/10.21037/atm-20-1152). The authors have no conflicts of interest to declare.

Ethical Statement: The authors are accountable for all aspects of the work in ensuring that questions related to the accuracy or integrity of any part of the work are appropriately investigated and resolved. The study was conducted in accordance with the Declaration of Helsinki (as revised in 2013). The study was approved by institutional ethics board of the fourth affiliated hospital of Harbin Medical University (No.: 2020-SCILLSC-07) and informed consent was taken from all the patients.

Open Access Statement: This is an Open Access article distributed in accordance with the Creative Commons Attribution-NonCommercial-NoDerivs 4.0 International License (CC BY-NC-ND 4.0), which permits the noncommercial replication and distribution of the article with the strict proviso that no changes or edits are made and the original work is properly cited (including links to both the formal publication through the relevant DOI and the license). See: https://creativecommons.org/licenses/by-nc-nd/4.0/.

\section{References}

1. Taylor PN, Albrecht D, Scholz A, et al. Global epidemiology of hyperthyroidism and hypothyroidism. Nat Rev Endocrinol 2018;14:301-16.

2. Zimmermann MB, Jooste PL, Pandav CS. Iodinedeficiency disorders. Lancet 2008;372:1251-62.

3. Shan Z, Chen L, Lian X, et al. Iodine Status and Prevalence of Thyroid Disorders After Introduction of Mandatory Universal Salt Iodization for 16 Years in China: A Cross-Sectional Study in 10 Cities. Thyroid 2016;26:1125-30.

4. Sun X, Shan Z, Teng W. Effects of increased iodine intake on thyroid disorders. Endocrinol Metab (Seoul) 
2014;29:240-7.

5. Weng $W$, Dong $M$, Zhan J, et al. A PRISMA-compliant systematic review and meta-analysis of the relationship between thyroid disease and different levels of iodine intake in mainland China. Medicine (Baltimore) 2017;96:e7279.

6. Guth S, Theune U, Aberle J, et al. Very high prevalence of thyroid nodules detected by high frequency $(13 \mathrm{MHz})$ ultrasound examination. Eur J Clin Invest 2009;39:699-706.

7. Tomimori E, Pedrinola F, Cavaliere H, et al. Prevalence of incidental thyroid disease in a relatively low iodine intake area. Thyroid 1995;5:273-6.

8. Woestyn J, Afschrift M, Schelstraete K, et al. Demonstration of nodules in the normal thyroid by echography. Br J Radiol 1985;58:1179-82.

9. Coltrera MD. Clinician-performed thyroid ultrasound. Otolaryngol Clin North Am 2014;47:491-507.

10. Tessler FN, Middleton WD, Grant EG, et al. ACR Thyroid Imaging, Reporting and Data System (TI-RADS): White Paper of the ACR TI-RADS Committee. J Am Coll Radiol 2017;14:587-95.

11. Kim DW, Eun CK, In HS, et al. Sonographic differentiation of asymptomatic diffuse thyroid disease from normal thyroid: a prospective study. AJNR Am J Neuroradiol 2010;31:1956-60.

12. Yeh HC, Futterweit W, Gilbert P. Micronodulation: ultrasonographic sign of Hashimoto thyroiditis. J Ultrasound Med 1996;15:813-9.

13. Pedersen OM, Aardal NP, Larssen TB, et al. The value of ultrasonography in predicting autoimmune thyroid disease. Thyroid 2000;10:251-9.

14. Song J, Zou SR, Guo CY, et al. Prevalence of Thyroid

Cite this article as: Miao K, Zhang L, Dong X, Qi Y, Kong D, Liu Y, Zhao N, Hu J. Iodine nutrition and prevalence of sonographic thyroid findings in adults in the Heilongjiang Province, China. Ann Transl Med 2020;8(21):1439. doi: 10.21037/ atm-20-1152
Nodules and Its Relationship with Iodine Status in Shanghai: a Population-based Study. Biomed Environ Sci 2016;29:398-407.

15. Yan Y, Liu S, Liu Y, et al. Investigations of iodinenutritional status of school-aged children in Harbin city. Chinese Journal of Control of Endemic Disenaces 1995;4:206-8.

16. König F, Andersson M, Hotz K, et al. Ten repeat collections for urinary iodine from spot samples or 24hour samples are needed to reliably estimate individual iodine status in women. J Nutr 2011;141:2049-54.

17. Vitamin and mineral requirements inhuman nutrition. Geneva, Switzerland: World Health Organization. Available online: https://www.who.int/nutrition/ publications/micronutrients/9241546123/en/

18. Ministry of Health. Announcement on food safety national standard "iodine content in edible salt" 2011. Available online: http://www.moh.gov.cn/publicfiles/business/ htmlfiles/mohwsjdj/s7891/201109/53064.htm. Accessed December 12, 2017.

19. Zou S, Wu F, Guo C, et al. Iodine nutrition and the prevalence of thyroid disease after salt iodization: a crosssectional survey in Shanghai, a coastal area in China. PLoS One 2012;7:e40718.

20. Guo Y, Zynat J, Xu Z, et al. Iodine nutrition status and thyroid disorders: a cross-sectional study from the Xinjiang Autonomous Region of China. Eur J Clin Nutr 2016;70:1332-6.

21. Wang N, Fang H, Fu C, et al. Associations of adiposity measurements with thyroid nodules in Chinese children living in iodine-sufficient areas: an observational study. BMJ Open 2017;7:e016706. 